\title{
Anisotropic viscous dissipation in three-dimensional magnetic merging solutions
}

\author{
I. J. D. Craig and Y. E. Litvinenko
}

Department of Mathematics, University of Waikato, PB 3105, Hamilton, New Zealand

Received 4 February 2009 / Accepted 23 April 2009

\begin{abstract}
Aims. We consider viscous and resistive energy dissipation in the flaring solar corona.

Methods. We compute energy dissipation rates, associated with magnetic merging in three dimensions. We examine an exact 3D solution for steady magnetic merging in a viscous resistive incompressible plasma. We use the Braginskii stress tensor to model viscous effects and derive scalings for the resistive and viscous dissipation rates. We evaluate the dissipation rates for typical parameters of solar active regions.

Results. Large-scale advective flows required to sustain resistive current sheets are shown to be associated with significant viscous losses. For plausible dimensionless resistivities (inverse Lundquist numbers), whether classical $\eta \leq 10^{-14}$ or anomalous $\eta \leq 10^{-8}$, viscous loss rates greatly exceed the resistive loss rates of the current layer and can approach flare-like rates of $10^{28} \mathrm{erg} \mathrm{s}^{-1}$.
\end{abstract}

Key words. magnetohydrodynamics (MHD) - sun: flares - sun: magnetic fields

\section{Introduction}

One of the outstanding problems in astrophysics is explaining the explosive energy release of solar and stellar flares. Although magnetic reconnection is the accepted release mechanism, the weak electrical resistivity in the solar corona leads to energyloss rates that are generally too slow to account for flare observations (Priest \& Forbes 2000). To what extent the presence of other physical ingredients - such as Hall effects - can increase the reconnection rate is currently the focus of intense theoretical investigation (e.g., Birn et al. 2001; Knoll \& Chacon 2006; Litvinenko 2009 and references therein).

A common element of virtually all reconnection models is the presence of strong Alfvénic exhaust flows. Observational studies (e.g., McKenzie \& Hudson 1999; Asai et al. 2004) indeed confirm that strong outflows are correlated with impulsive hard X-ray bursts in flares. Since typical merging models also require strong advective inflows to carry magnetic field lines into the reconnection region, it is natural to ask: How significant are the viscous energy losses that derive from velocity fields associated with magnetic merging? It is this question that motivates the present study.

As background, we note that Hollweg $(1985,1986)$ has already argued that viscous damping is likely to dominate resistive dissipation under a wide range of coronal conditions. In particular, because coronal plasmas are only weakly resistive, huge gradients in the magnetic field - typically requiring near-singular current sheets - are required to provide appreciable Ohmic dissipation rates. By contrast, viscous dissipation can achieve significant rates with relatively modest gradients in the velocity field of the plasma. This point has recently been emphasized by two planar reconnection studies, namely, the incompressible steady merging model of Litvinenko (2005) and the compressible $X$-point collapse analysis of Craig (2008).
Consider, for instance, a coronal velocity field of global scale $l_{\mathrm{c}}$. In the case of Alfvénic speeds the vorticity will scale as $v_{\mathrm{A}} / l_{\mathrm{c}}$ and therefore the global viscous losses can be estimated as

$W_{v} \simeq v v_{\mathrm{A}}^{2} l_{\mathrm{c}}$.

Taking a classical shear viscosity $v$ for the corona and assuming values $l_{\mathrm{c}}=10^{9.5} \mathrm{~cm}$ and $v_{\mathrm{A}}=10^{9} \mathrm{~cm} \mathrm{~s}^{-1}$, appropriate to a coronal temperature of $2 \times 10^{6} \mathrm{~K}$ (see Sect. 2.1), yields the estimate $W_{v} \simeq 10^{27} \mathrm{erg} \mathrm{s}^{-1}$. This dissipation rate is already comparable to the output of a modest flare.

It would be a major deception, however, to apply an estimate based on Eq. (1) to magnetic merging in the solar corona. This is because an accurate treatment of viscous dissipation in a magnetically stratified plasma requires a tensor description that effectively suppresses shear viscosity components (Braginskii 1965). In the case of planar merging driven by a stagnation point flow, the bulk viscosity provides dissipation rates that are comparable to those based on the classical shear viscosity (Litvinenko 2005). But if an axial field, rather than a planar field, were advected by the flow, the viscous dissipation would be entirely negligible (see Sect. 2.2).

These considerations suggest that dissipation due to bulk viscosity may depend in a complicated way on the details of the field geometry. In the present paper we extend recent studies that incorporate bulk viscosity within planar magnetic geometries (Litvinenko 2005; Craig 2008). Specifically we analyze the resistive and viscous dissipation provided by an exact steady three-dimensional (3D) magnetohydrodynamic (MHD) solution for incompressible magnetic merging (Craig \& Watson 2000). We also generalize our previous calculation (Litvinenko 2005) by determining the effect of the magnetic field pile-up at the entrance to the reconnecting current sheet on the resistive and viscous dissipation rates.

The incompressible MHD equations are introduced in Sect. 2 where we discuss energy losses due to resistive and viscous 
effects under typical coronal conditions. Our main results are derived in Sect. 3 where we introduce a 3D "fan" merging solution, based on an axisymmetric velocity field. This model is used to provide concrete scaling laws for the resistive and viscous losses. In Sect. 4 we present our conclusions.

\section{Viscous and resistive dissipation in magnetized coronal plasmas}

\subsection{Introduction}

Our aim is to construct a three-dimensional magnetic merging solution that allows us to evaluate the global viscous and resistive losses. To do this we work with the incompressible MHD equations, scaled with respect to typical solar coronal values for field strength $B_{\mathrm{c}}=10^{2} \mathrm{G}$, size scale $l_{\mathrm{c}}=10^{9.5} \mathrm{~cm}$, and number density $n_{\mathrm{c}}=10^{9} \mathrm{~cm}^{-3}$. Times are measured in units of $l_{\mathrm{c}} / v_{\mathrm{A}}$ where $v_{\mathrm{A}} \simeq 10^{9} \mathrm{~cm} \mathrm{~s}^{-1}$ is the Alfvén speed. The global energy loss rate has the units $B_{\mathrm{c}}{ }^{2} /(8 \pi) v_{\mathrm{A}} l_{\mathrm{c}}{ }^{2} \simeq 4 \times 10^{30} \mathrm{erg} \mathrm{s}^{-1}$.

Energy losses from the source volume are controlled by two small parameters, the dimensionless resistivity $\eta^{\star}$ and the dimensionless plasma viscosity $v^{\star}$. For a collisional plasma of temperature $T=10^{6} \mathrm{~K}$ with conductivity $\sigma \sim T^{3 / 2}$ (Spitzer 1962), $\eta^{\star}$ is an inverse Lundquist number of magnitude

$\eta^{\star}=\frac{c^{2}}{4 \pi v_{\mathrm{A}} l_{\mathrm{c}} \sigma} \simeq 10^{-14.5}$

Viscous losses are determined by an inverse of the traditional Reynolds number. For a plasma of mass density $\rho_{\mathrm{c}}$ in which the viscosity scales as $v \sim T^{5 / 2}$ (Spitzer 1962), we find the following value for the dimensionless viscosity coefficient:

$v^{\star}=\frac{v}{\rho_{\mathrm{c}} v_{\mathrm{A}} l_{\mathrm{c}}} \simeq 10^{-4.5}$

From now on, we work only with dimensionless quantities, so we drop the superscripts and use simply $v$ and $\eta$.

The fact that $v \gg \eta$ suggests that viscous dissipation is likely to dominate resistive damping for typical coronal plasmas. This dominance can be expected to increase in active region plasmas where plasma temperatures can exceed several million degrees. We should remember, however, that energy that derives from the topological simplification of the magnetic field can only be released resistively via magnetic reconnection. A further complication is that viscous effects become highly anisotropic in magnetized coronal plasmas (Braginskii 1965; Hollweg 1986). Isotropy breaks down when the proton mean free path exceeds the gyroradius: $\omega_{\mathrm{p}} \tau_{\mathrm{p}} \gg 1$, where $\omega_{\mathrm{p}}=\mathrm{e} B /\left(m_{\mathrm{p}} c\right)$ is the proton cyclotron frequency and $\tau_{\mathrm{p}} \simeq 0.75 T^{3 / 2} / n$ is the mean time (in seconds) between momentum-changing collisions. Typical coronal parameters lead to $\omega_{\mathrm{p}} \tau_{\mathrm{p}} \simeq 10^{6} \gg 1$, which confirms that anisotropic viscosity must be used in almost all coronal applications.

\subsection{Incompressible MHD equations}

We adopt the 3D incompressible MHD equations for the velocity field $\boldsymbol{v}(\boldsymbol{r}, t)$ and the magnetic field $\boldsymbol{B}(\boldsymbol{r}, t)$. These comprise
Maxwell's equations and the momentum and continuity equations:

$$
\begin{aligned}
\partial_{t} \boldsymbol{v}+\boldsymbol{v} \cdot \nabla \boldsymbol{v} & =\boldsymbol{J} \times \boldsymbol{B}-\nabla P+\nabla \cdot \mathcal{S} \\
\partial_{t} \boldsymbol{B} & =\nabla \times(\boldsymbol{v} \times \boldsymbol{B})-\eta \nabla \times \boldsymbol{J} \\
\nabla \cdot \boldsymbol{B} & =\nabla \cdot \boldsymbol{v}=0
\end{aligned}
$$

Here $P$ is the plasma pressure, $\nabla \cdot \mathcal{S}$ is the viscous force and $\boldsymbol{J}=\nabla \times \boldsymbol{B}$ the current density.

As already mentioned, the classical expression for the viscous tensor $\mathcal{S}$ in an incompressible fluid, namely

$\mathcal{S}_{i j}=v\left(\partial_{j} v_{i}+\partial_{i} v_{j}\right)$

is not accurate for a magnetized collisional plasma in which the proton mean free path greatly exceeds the proton gyroradius. In such cases the strong field form (Braginskii 1965) is appropriate:

$\mathcal{S}_{i j}=v_{0}\left(3 \frac{B_{i} B_{j}}{B^{2}}-\delta_{i j}\right)\left(\frac{B_{m} B_{k}}{B^{2}} \partial_{k} v_{m}\right)$.

Here $v_{0} \simeq v$ (Hollweg 1985) and summation over repeated suffixes is assumed. As noted above, a planar flow that advects a purely axial field yields $\mathcal{S}=0$. More generally, it is clear from Eq. (8) that $\mathcal{S}$ vanishes if $(\boldsymbol{B} \cdot \nabla) \boldsymbol{v}=0$.

For completeness, we should mention that the stress tensor $\mathcal{S}$ generally contains an extra term $\sim \nabla \cdot v$ that accounts for the finite compressibility of the plasma. This term provides an avenue for bulk viscous dissipation (Sect. 3.5) that is not represented in the present incompressible analysis.

\subsection{Global energy losses}

Before considering explicit solutions, it is instructive to obtain a general expression for the global energy losses, valid for all forms of viscosity. To do this we dot the primitive momentum Eq. (4) with $\boldsymbol{v}$ and permute $\boldsymbol{v} \cdot[(\nabla \times \boldsymbol{B}) \times \boldsymbol{B}]$, making use of the identity

$$
\begin{aligned}
\nabla \cdot[\boldsymbol{B} \times(\boldsymbol{v} & \times \boldsymbol{B})] \\
& =(\boldsymbol{v} \times \boldsymbol{B}) \cdot \nabla \times \boldsymbol{B}-\boldsymbol{B} \cdot \nabla \times(\boldsymbol{v} \times \boldsymbol{B}) .
\end{aligned}
$$

Using the induction equation to re-express the final term we find that

$$
\begin{aligned}
\partial_{t} \frac{1}{2}\left(v^{2}+B^{2}\right) & \\
+\nabla \cdot\left(\left(P+\frac{1}{2} v^{2}\right) \boldsymbol{v}+\boldsymbol{B}\right. & \times(\boldsymbol{v} \times \boldsymbol{B})+\eta \boldsymbol{J} \times \boldsymbol{B}-\mathcal{S} \cdot \boldsymbol{v}) \\
& =-\eta J^{2}-\mathcal{S}: \nabla \boldsymbol{v}
\end{aligned}
$$

where $\mathcal{S}: \nabla \boldsymbol{v} \equiv \mathcal{S}_{i j} \partial_{j} v_{i}$

In what follows we are interested in steady state solutions $\left(\partial_{t}=0\right)$ in a volume $V$, sustained by advective flows through the boundary. In this case the resistive and viscous losses (the right-hand side terms in Eq. (10)) can be integrated over $V$ and the divergence term evaluated as a surface contribution. We see that energy dissipation in a steady solution must be balanced by the Poynting flux and the work done by viscous stresses. 


\section{The visco-resistive merging solution}

\subsection{Merging solutions in $3 D$}

It is now recognised that $3 \mathrm{D}$ magnetic merging at an isolated null requires the presence of highly localized current structures in the form of current sheets or quasi-cylindrical current tubes. Such structures account for resistive dissipation in so-called "fan" and "spine" reconnection models. More specifically, fan solutions can be derived using a form

$\boldsymbol{B}=[X(z), Y(z), 0]$

that defines a current sheet in the fan plane $z=0$. Spine solutions, in which $\boldsymbol{B}=Z(x, y) \hat{z}$, have a considerably richer current structure (Craig \& Fabling 1996). Currents are localized about the $z$-axis (the "spine"). Due to the strong localization, however, spine models are generally less effective than fan models for rapid energy release. But no matter whether fan or spine merging is considered, the unknown components of the field are determined from the induction Eq. (5), using a specified flow solution.

\subsection{Velocity field for fan merging}

We now ask: what kind of flows can support a steady fan merging solution? Note that the fan current structure $\boldsymbol{J}=$ $\left[-Y^{\prime}(z), X^{\prime}(z), 0\right]$ leads to a Lorentz force that is irrotational. This implies that any velocity field that satisfies the curled form of the momentum Eq. (4), namely

$\nabla \times(\boldsymbol{v} \cdot \nabla) \boldsymbol{v}-\nabla \times(\nabla \cdot \mathcal{S})=0$

provides a possible solution.

Now, from Craig \& Watson (2000), we know that

$\boldsymbol{v}=\alpha\left[\frac{\omega}{2}(x \cos \omega z+y \sin \omega z)\right.$,

$$
\left.\frac{\omega}{2}(y \cos \omega z-x \sin \omega z),-\sin \omega z\right],
$$

provides an exact, axisymmetric solution in the absence of viscous effects. Here the parameter $\alpha>0$ determines the strength of the flow, and $\omega$ is a wavenumber in the range $0<\omega<\pi$. Using this form, together with Eq. (11), we find (dropping the subscript on $v_{0}$ ) that

$$
\mathcal{S}=\frac{v \alpha \omega}{2} \cos (\omega z)\left(\begin{array}{ccr}
3 X^{2} / B^{2}-1 & 3 X Y / B^{2} & 0 \\
3 X Y / B^{2} & 3 Y^{2} / B^{2}-1 & 0 \\
0 & 0 & -1
\end{array}\right) .
$$

The Braginskii tensor retains only a z-dependence and a simple check shows that $\nabla \times \nabla \cdot \mathcal{S}$ vanishes. The consistency requirement (12) is therefore satisfied, and so Eq. (13) provides an exact analytical description of visco-resistive magnetic merging in 3D.

The solution is completed by solving Eq. (5) for the magnetic field components:

$$
\begin{aligned}
& \eta X^{\prime \prime}+\alpha \sin (\omega z) X^{\prime}+\frac{\alpha \omega}{2}[\cos (\omega z) X+\sin (\omega z) Y]=0, \\
& \eta Y^{\prime \prime}+\alpha \sin (\omega z) Y^{\prime}+\frac{\alpha \omega}{2}[\cos (\omega z) Y-\sin (\omega z) X]=0 .
\end{aligned}
$$

This system, first derived by Craig \& Watson (2000), describes the merging of magnetic field lines, washed in through the planes $z= \pm 1$. The dimensionless inflow speed at $z= \pm 1$ is given by $\alpha \sin \omega$. Field lines are rotated about the $z$-axis as they are advected towards the current layer centered on the fan plane

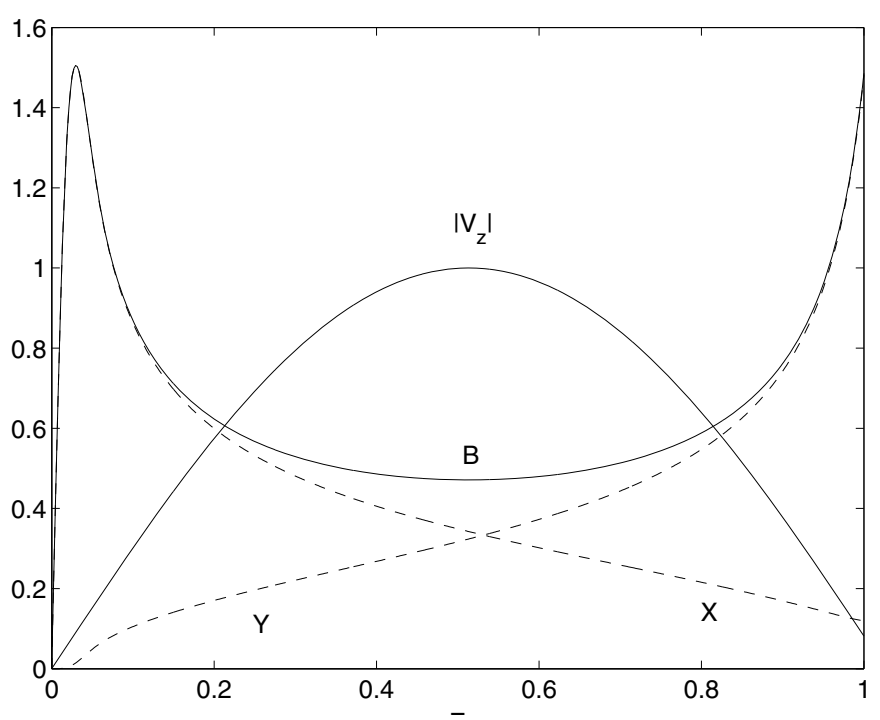

Fig. 1. Inflow magnetic field and flow speed against $z$ for the parameters $\eta=0.001, \alpha=1, \omega=3$. The amplitude $B_{\mathrm{s}}$ of the total field $B$ is set by the exhaust speed of material ejected in the $z=0$ fan plane. Note that the $x$-component of the field provides the main contribution to the current layer.

$z=0$, where they are resistively dissipated. What differs from Craig \& Watson (2000) when $\mathcal{S} \neq 0$ is the requirement that the external viscous stresses compensate for the viscous losses in the volume and maintain the steady state solution (see Eq. (10)).

\subsection{Computed merging solutions}

The solution outlined above is exact, but to obtain plausible dissipation rates we must ensure that magnetic field is coupled to the flow in a physically realistic manner. In practice, this can be achieved by matching the magnetic pressure in the current sheet to the dynamic pressure of the plasma exhaust-a prescription that provides, for a specified peak field in the current layer $B_{\mathrm{s}}$, a well defined merging rate (Litvinenko \& Craig 1999, 2000; Craig \& Watson 2000). In the results below the peak field $B_{\mathrm{s}}$ is identified with the exhaust speed in the fan $(\alpha \omega / 2)$ at unit radial distance from the origin. The solution is obtained by "shooting" using Eqs. (15) and (16), adjusting gradients at the origin until the required field amplitude is achieved over the inflow region.

Figure 1 shows a typical solution plotted along the inflow $z$-axis for the case $\eta=0.001, \alpha=1, \omega=3$. The solution is dominated by a large scale advective region but there is a narrow current layer overlying the origin that accounts for the bulk of the Ohmic dissipation. Here we have modeled the current layer by taking $Y^{\prime}(0)=0$ and varying $X^{\prime}(0)$ in the numerical integration of Eqs. (15) and (16). (Note for clarity that the fact $Y^{\prime}(0)=0$ is obscured in Fig. 1 by the strong variation in the solution close to the origin). The $X$-field component is therefore dominant in the region $0 \leq z<0.5$, but this dominance is lost in regions approaching the outer boundary. It is also possible to tune the wavenumber so that $B( \pm 1) \simeq B_{\text {s. }}$ This adjustment is minor (a few percent) and does not impact significantly on the computed dissipation rates.

In view of the smallness of the coronal resistivity, in practice it is important to understand how the solution behaves over an extended range of $\eta$. In Fig. 2 we plot the global resistive and viscous dissipation rates over the resistive range $10^{-7}<\eta<10^{-2}$ 


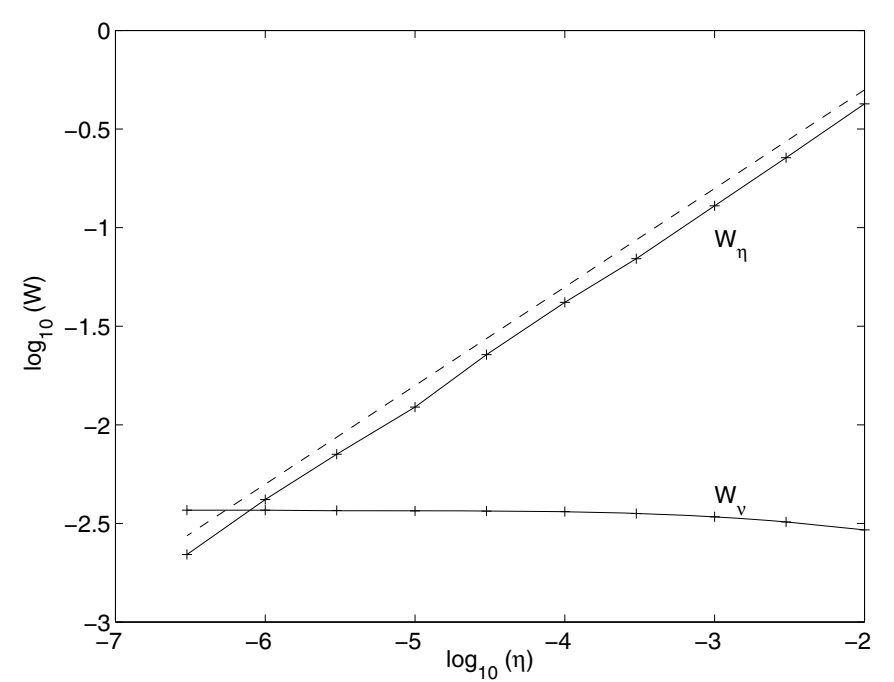

Fig. 2. Scaling of resistive and viscous dissipation rates over the resistive range $10^{-7}<\eta<10^{-2}$. The Braginskii viscous losses, based on $v=10^{-3}$, are effectively independent of the resistivity and dominate in the regime $\eta<10^{-6}$. The dotted reference line indicates the resistive scaling $5 \eta^{1 / 2}$. Note that all these rates refer to one quadrant, say $x>0, y>0$, of the merging geometry.

for the flow strength $\alpha=1$. We obtain the global dissipation rates by integrating the volumetric rates, defined by Eq. (10): $W_{\eta}=\int \eta J^{2} \mathrm{~d} V$ and $W_{v}=\int \mathcal{S}: \nabla \boldsymbol{v} \mathrm{d} V$. The dimensionless Braginskii viscosity coefficient was fixed at $v=10^{-3}$, which corresponds to an active region temperature of around $4 \times 10^{6} \mathrm{~K}$. With these parameters we see that the viscous losses exceed the resistive losses in the physically relevant regime $\eta<10^{-7}$. Note that, although $W_{\eta}$ approximates an $\eta^{1 / 2}$ scaling, $W_{v}$ is effectively independent of the resistivity. The calculated rate $W_{v} \simeq 10^{-2.5}$ corresponds to an energy loss rate exceeding $10^{28} \mathrm{erg} \mathrm{s}^{-1}-$ and such rates can legitimately be multiplied by four since only one quadrant, say $x>0, y>0$, of the full merging geometry has been considered for the integration.

As we argued previously (Litvinenko \& Craig 1999, 2000), although any solution is specified by four input parameters $-\eta$, $v, \alpha$ and $\omega$ - two physically based restrictions should be imposed on the model: first, the exhaust speed of the flow should be comparable to the Alfvén speed based on the peak magnetic field $B_{\mathrm{s}}$; second, the field $B_{\mathrm{s}}$ in the current sheet must saturate at some level $B_{\mathrm{s}}=B_{\mathrm{s}}^{*}$, independent of the values of small dissipation parameters $\eta$ and $v$. We refer to these as the equalization $(i)$ and saturation ( $i$ i) conditions. These conditions lead to the maximum achievable dissipation rates and thus allow us to test the viability of the model in application to flare-like energy release.

Although condition $(i)$ is built into the numerical results, the absence of condition (ii) suggests that dissipation rates could be increased indefinitely by allowing larger values of the sheet field $B_{\mathrm{s}}$. By taking an upper limit for $B_{\mathrm{S}}$ we can restrict both the merging rate and the exhaust flow to physically plausible levels. More generally, by applying conditions ( $i$ ) and (ii) systematically, the dependence of the dissipation rates on $\alpha$ and $\omega$ can be effectively eliminated: in this case the scalings are specified by $\eta$ and $v$ and the saturated peak field strength $B_{\mathrm{s}}^{*}$.

\subsection{Resistive and viscous scalings}

Consider first the Ohmic dissipation rate $W_{\eta}$. Since the merging solution always comprises an outer advection region $z>z_{\mathrm{s}}$ and a narrow resistive current layer $z<z_{\mathrm{s}} \ll 1$ we can write

$W_{\eta}=\int \eta J^{2} \mathrm{~d} V \simeq \eta \frac{B_{\mathrm{s}}{ }^{2}}{z_{\mathrm{s}}}$.

In order to estimate the resistive layer thickness $z_{\mathrm{s}}$, note that both the $X$ and $Y$ components of the magnetic field are significant in the advection region but the resistive dissipation is negligible. By setting $\eta=0$ in Eqs. (15) and (16) and combining the two equations into a single one for the energy density $B^{2}=X^{2}+Y^{2}$, it is easy to show that the quantity $\mathcal{F} \equiv B^{2} \sin (\omega z)$ is conserved. Evaluating $\mathcal{F}$ at the inflow boundary $z=1$ and at the onset of the sheet $z \simeq z_{\mathrm{s}}$ gives

$B_{1}^{2} \sin \omega \simeq \omega z_{\mathrm{s}} B_{\mathrm{s}}^{2}$,

assuming $z_{\mathrm{s}} \ll 1$. Equation (18) yields an expression for $z_{\mathrm{s}}$ in terms of $\omega, B_{\mathrm{s}}$ and the boundary field $B_{1}$, which is explored below (Fig. 3).

The properties of the current layer can be specified using the equalization and saturation conditions, introduced in the previous section. The equalization condition $(i)$ follows from the form of the solution (13) for the plasma flow:

$\alpha \omega \simeq B_{\mathrm{s}}$.

In the resistive layer we can assume-by a suitable orientation of the magnetic field on the inflow boundary-that the $X$ component of the field is dominant. Since the inflow is approximately linear in the region $z<z_{\mathrm{s}}$ we obtain a Kummer equation for $X$ :

$\frac{\eta}{\alpha \omega} X^{\prime \prime}+z X^{\prime}+\frac{1}{2} X=0$

The important point is the scaling of the current sheet thickness

$z_{\mathrm{s}} \simeq \sqrt{\frac{\eta}{\alpha \omega}} \simeq \sqrt{\frac{\eta}{B_{\mathrm{s}}}}$.

Eliminating $\alpha, \omega$, and $z_{\mathrm{s}}$ using the expressions above and replacing $B_{\mathrm{s}}$ by its saturated value $B_{\mathrm{s}}^{*}$ leads to the result:

$W_{\eta} \simeq \eta^{1 / 2} B_{\mathrm{s}}^{* 5 / 2}$.

If necessary, Eqs. (18) and (21) can be solved to give the values of $\alpha$ and $\omega$ in terms of $B_{\mathrm{s}}^{*}$ and $\eta$ in the optimized saturated solution.

Now consider the bulk viscosity dissipation $W_{v}$. Using Eqs. (13) and (14), we find that

$\mathcal{S}: \nabla \boldsymbol{v}=\frac{3}{4} v \alpha^{2} \omega^{2} \cos ^{2}(\omega z)$

For $\omega \simeq 3$ (as in Fig. 1) the volumetric viscous heating is maximum at the origin, falls to zero when $z \simeq 1 / 2$, and increases towards the outer boundary. It follows that

$W_{v}=\int \mathcal{S}: \nabla v \mathrm{~d} V=\frac{3}{8} v \alpha^{2} \omega^{2}\left(1+\frac{\sin (2 \omega)}{2 \omega}\right)$.

Since the bracketed term is of order unity in the interval $\omega<$ $\pi$, we can immediately obtain the sought-after result, using the saturation condition $\alpha \omega=B_{\mathrm{s}}$ and ignoring a numerical factor of order unity:

$W_{v} \simeq v B_{\mathrm{s}}^{* 2}$. 


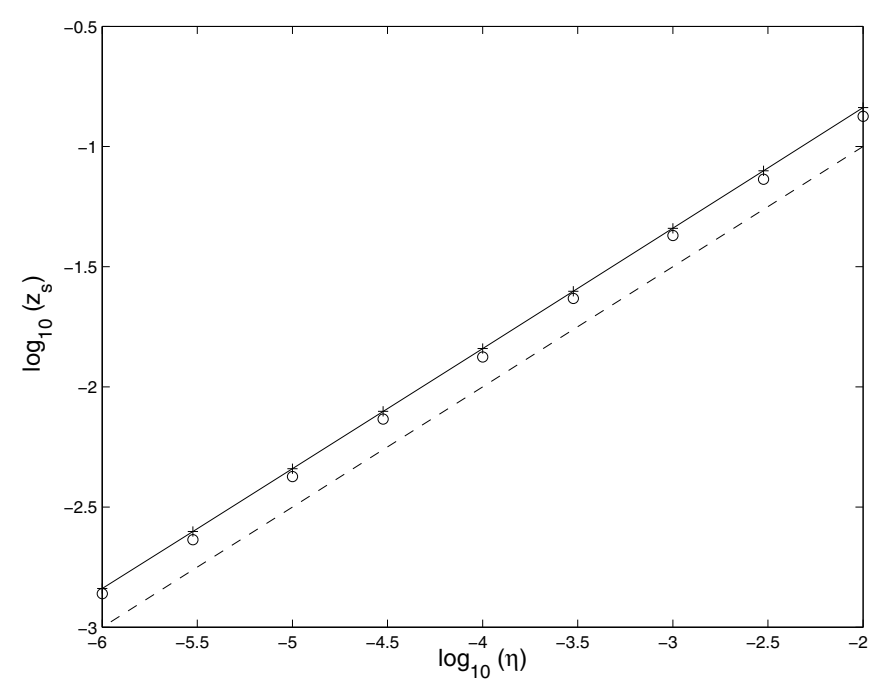

Fig. 3. Current sheet thickness based on measured position of the peak field (circles) against location (solid line with crosses) estimated using formula (18). Results are computed for $\omega=1.5, B_{\mathrm{s}}=1$, using measured values of boundary field $B_{1}$. The dotted line shows $z_{\mathrm{s}}=\eta^{1 / 2}$ scaling.

The dependence of $W_{\eta}$ and $W_{v}$ on the pile-up factor $B_{\mathrm{s}}^{*}$ is a new result that is also valid for other saturated and optimized merging solutions. Note that $B_{\mathrm{s}}^{*} \simeq 1$ was assumed in a previous analysis of visco-resistive 2D merging (Litvinenko 2005).

We see that

$\frac{W_{\eta}}{W_{v}} \simeq \frac{\eta^{1 / 2}}{v} B_{\mathrm{s}}^{* 1 / 2}$

is likely to be $10^{-1}$ or smaller for any plausible resistivity $\eta<10^{-8}$. While large saturated fields $B_{\mathrm{s}}^{*}>5$ can significantly enhance dissipation rates, they are unlikely to undo the overall dominance of the viscous energy losses. The weak dependence $\sim B_{\mathrm{s}}^{* 1 / 2}$ means that the conclusions in our previous study (Litvinenko 2005) remain valid for all plausible values of $B_{\mathrm{s}}^{*}$.

Finally we comment on the accuracy of the analytical scalings above. Figure 3 shows a comparison of $z_{\mathrm{s}}$, defined by the position of the peak field $B_{\mathrm{s}}$, against the approximate formula $B_{1}^{2} \sin \omega /\left(\omega B_{\mathrm{s}}^{2}\right)$ of Eq. (18). The relative agreement is better than ten percent over a range of resistivities $10^{-6} \leq \eta \leq 10^{-2}$. Thus the boundary layer arguments used to derive the scaling laws outlined above are remarkably well supported by the numerics.

\subsection{Discussion}

The present results suggest that viscous losses are likely to dominate resistive losses in magnetic merging solutions for all plausible plasma resistivities. The possibility that an enhanced "anomalous" resistivity can nullify this conclusion seems remote given that enhancements of order $10^{6}$ are the most that can be expected (e.g., Litvinenko \& Craig 2000 and references therein). Even for $\eta$ as large as $10^{-8}, W_{\eta}$ can exceed $W_{v}$ only if $v \simeq 10^{-4}$ lies at the lower end of its plausible range in the solar corona.

Possibly the main limitation of the present analysis is the requirement of a global vortical flow to drive the merging. Clearly, on physical grounds, the velocity field should fall off substantially outside the solution domain $r^{2}=x^{2}+y^{2} \leq 1$. Were this not the case then unphysically large hydro-magnetic pressures would be required to sustain the merging. Whether large-scale velocity fields with the required properties are likely to develop in the active solar corona - say, as a result of flux emergence or filament eruption - is far from assured. Yet it should be kept in mind that the present model represents only one solution in a range of theoretical possibilities.

Consider, for example, an $X$-point implosion within a closed planar geometry. Reconnection is driven by a rapid localization in both the $\boldsymbol{B}$ and $\boldsymbol{v}$ fields, and viscous dissipation is enhanced by strong compressive effects that accompany the collapse (Craig 2008). This physical picture is quite different from that of the present model. Specifically, the present solution does not rely on small length scales in the velocity field in order to achieve significant bulk viscous losses. Taking both models together, however, strongly suggests that anisotropic viscous effects can lead to flare-like energy release rates in a variety of magnetic merging geometries.

\section{Conclusions}

The present study has addressed the viscous and resistive energy losses associated with an exact incompressible magneticmerging solution. We have shown that large-scale advective flows, required to sustain 3D "fan" merging at an isolated neutral point, are subject to significant viscous energy losses based on the bulk viscosity terms in the Braginskii viscous stress tensor. Specifically, for typical coronal parameters, the viscous dissipation rate can reach flare-like levels of $10^{28} \mathrm{erg} \mathrm{s}^{-1}$. This rate dominates resistive losses for all plausible resistivities, whether classical $\eta \simeq 10^{-14}$ or turbulent $\eta \simeq 10^{-8}$.

One curious feature of the present analysis is that the global viscous losses seem almost independent of the rate of magnetic merging. Certainly, when the peak magnetic field strength is fixed, the viscous loss rate is independent of the resistivity (Fig. 2). However, for stronger field strengths $B_{\mathrm{s}}$, both the dynamic pressure of the plasma flow and the viscous loss rate are enhanced, $W_{v} \simeq v B_{\mathrm{s}}^{2}$.

Notably absent in the present solution is a small length scale controlled by the plasma viscosity. For example, a hybrid length scale $(v \eta)^{1 / 4}$ is known to emerge in other reconnection solutions, for example, those based on $X$-point collapse, for both classical and anisotropic viscosities (Craig et al. 2005; Craig 2008). Although these compressible solutions are very different from the models considered here (see Sect. 3.5), they are consistent with fast viscous dissipation, driven by strong localizations in the velocity field, at least in the regime $v \gg \eta$. What the present analysis confirms is that alternative solutions are possible in which strong bulk viscous losses are not dependent on the development of small length scales in the velocity field that drives the merging.

Our approach is limited in two respects, justifying future research. First, we explored the viability of the 3D magnetic merging model by calculating the maximum energy release rate, consistent with physical requirements of optimization and saturation of the solution. Flare observations should be used to investigate whether the large-scale vortical flows, required to sustain the maximum viscous dissipation rate, are indeed present in the flaring solar corona. Second, we have used the leading bulk viscosity term of the Braginskii viscous stress tensor in our analysis. Because the expression is valid in a strong field limit, our analysis cannot be extended to the neighborhood of a magnetic null. This is not critical in the present analysis because the viscous stress tensor is valid everywhere except for a thin strip $z \ll z_{\mathrm{s}} \simeq \sqrt{\eta}$ of negligible measure, which resides well inside the current sheet. However, a more general form of the viscous stress tensor, valid for arbitrary magnetic field 
strengths, should be employed to generalize our results. One possible choice would be the so-called Liley form of the stress tensor (e.g., Hosking \& Marinoff 1973), although it is not clear whether analytical treatment would be possible in that case.

Acknowledgements. This work was supported by NASA grant NNX08AG44G, NSF grants ATM-0734032 and ATM-0837915, and by a research fellowship from the Alexander von Humboldt Foundation. Discussions with Prof. Roger Hosking and a detailed, constructive report by an anonymous referee have been much appreciated. Joli Adams' encouragement to personalize the writing is acknowledged.

\section{References}

Asai, A., Yoshimoto, T., Shimojo, M., \& Shibata, K. 2004, ApJ, 605, L77 Birn, J., Drake, J. F., Shay, M. A., et al. 2001, J. Geophys. Res., 106, 3715
Braginskii, S. I. 1965, Rev. Plasma Phys. 1, 205

Craig, I. J. D. 2008, A\&A, 487, 1155

Craig, I. J. D., \& Fabling, R. B. 1996, ApJ, 462, 969

Craig, I. J. D., \& Watson, P. G. 2000, Solar Phys., 194, 251

Craig, I. J. D., Litvinenko, Y. E., \& Senanayake, T. 2005, A\&A, 433, 1139

Hollweg, J. V. 1985, J. Geophys. Res., 90, 7620

Hollweg, J. V. 1986, ApJ, 306, 730

Hosking, R. J., \& Marinoff, G. M. 1973, Plasma Phys., 15, 327

Knoll, D. A., \& Chacon, L. 2006, Phys. Rev. Lett., 96, 135001

Litvinenko, Y. E. 2005, Solar Phys., 229, 203

Litvinenko, Y. E. 2009, ApJ, 694, 1464

Litvinenko, Y. E., \& Craig, I. J. D. 1999, Solar Phys., 189, 315

Litvinenko, Y. E., \& Craig, I. J. D. 2000, ApJ, 544, 1101

McKenzie, D. E., \& Hudson, H. S. 1999, ApJ, 519, L93

Priest, E. R., \& Forbes, T. 2000, Magnetic reconnection: MHD theory and applications (Cambridge Univ. Press)

Spitzer, L. 1962, Physics of fully ionized gases (John Wiley \& Sons) 